\title{
Generating Estimates of Classification Confidence for a Case-Based Spam Filter
}

\author{
Sarah Jane Delany ${ }^{1}$, Pádraig Cunningham ${ }^{2}$, and Dónal Doyle ${ }^{2}$ \\ 1 Dublin Institute of Technology, \\ Kevin Street, Dublin 8, Ireland \\ sarahjane.delany@comp.dit.ie \\ 2 University of Dublin, Trinity College, \\ Dublin 2, Ireland \\ \{padraig.cunningham, donal.doyle\}@cs.tcd.ie
}

\begin{abstract}
Producing estimates of classification confidence is surprisingly difficult. One might expect that classifiers that can produce numeric classification scores (e.g. $k$-Nearest Neighbour or Naive Bayes) could readily produce confidence estimates based on thresholds. In fact, this proves not to be the case, probably because these are not probabilistic classifiers in the strict sense. The numeric scores coming from $k$-Nearest Neighbour or Naive Bayes classifiers are not well correlated with classification confidence. In this paper we describe a case-based spam filtering application that would benefit significantly from an ability to attach confidence predictions to positive classifications (i.e. messages classified as spam). We show that 'obvious' confidence metrics for a case-based classifier are not effective. We propose an ensemble-like solution that aggregates a collection of confidence metrics and show that this offers an effective solution in this spam filtering domain.
\end{abstract}

\section{Introduction}

One might expect that classifiers that produce numeric scores for class membership would deliver effective estimations of prediction confidence based on thresholds on these scores. Examples of classifiers that produce numeric scores in this manner are; Naive Bayes, $k$-Nearest Neighbour [1], Neural Networks [2], Logistic Regression [3] and Support Vector Machines [4]. Our experience with these classifiers suggests that the numeric scores from Support Vector Machines and Logistic Regression are predictive of confidence but those from Naive Bayes, Neural Networks and $k$-Nearest Neighbour $(k$-NN) are not. We demonstrate that this is the case for $k$-NN and Naive Bayes in Section 2.

In this paper we are concerned with generating estimates of classification confidence for a case-based spam filter called ECUE (Email Classification Using Examples) [5]. ECUE has the advantage of being very effective at tracking concept drift but this requires the user to identify False Positives (FPs) and False Negatives (FNs) so that they can be used to update the case-base. Identifying FNs is not a problem because they turn up in the Inbox (i.e. spam that has been 
allowed through the filter). Identifying FPs involves monitoring a spam folder to identify legitimate email that has been classified as spam. Our objective here is to be able to partition this class so that the user need only monitor a subset - the set for which the confidence is low.

A straightforward success criterion in this regard is the proportion of positives for which prediction confidence is high and the prediction is correct (clearly there cannot be any FPs in this set). A mechanism that could label more than $50 \%$ of the positive class (i.e. classified as spam) as confident and have no FPs in this set would be useful. The lower-confidence positives could be allowed into the Inbox carrying a Maybe-Spam marker in the header or placed in a Maybe-Spam folder that would be checked periodically.

In section 2 we provide a brief overview of research on estimating confidence. The basic indicators for confidence that can be used with $k$-NN are described in section 3 where we show that no single one of these measures is effective in estimating confidence. In section 4 we present some simple techniques for aggregating these basic indicators and present an evaluation on unseen data that shows a simple voting technique to be very effective. The paper concludes in section 5 with a summary and some suggestions for future work.

\section{Review}

Cheetham and Price have recently emphasised the importance of being able to attach confidence values to predictions in CBR [6,7]. This has been a research issue since the earliest days of expert systems research: it is part of the body of research on meta-level knowledge $[8,9]$, the view being that it is important for a system to 'know what it knows'. TEIRESIAS is a system in this spirit, it was designed to simply admit its ignorance instead of venturing risky advice [10].

More recently, the system SIROCCO from McLaren and Ashely [11] uses meta-rules to determine the system's confidence. Their system operates in an engineering ethics domain, in which incorrect suggestions could be considered sensitive and damaging. In this system, if any one of the meta-rules are fired then the system considers itself inadequate for the task. Their evaluation of SIROCCO shows that allowing the system to produce 'don't know' results reduces the number of incorrectly classified cases, with a small trade off whereby the number of correctly classified cases is reduced.

So while it is clear that it is useful to be able to produce estimates of confidence, it is also clear that that generating reliable estimates is not straightforward. Cheetham and Price [7] describe 12 measures of confidence that can be applicable for a $k$-NN classifier. Some of these indicators increase with confidence and some decrease. Since no single indicator is capable of producing a robust measure of confidence they explore the use of a decision tree, that is allowed to use all the measures, as a mechanism for aggregating all the available metrics. The authors show that, even using a decision tree to learn a good confidence measure from historic data, it is difficult to avoid the situation where predictions labelled as confident prove to be incorrect. They also emphasise that 
the confidence estimation mechanism will need to be updated over time as the nature of the problems being solved can change.

Because of this we choose to concentrate on simpler aggregation mechanisms. We engineered all indicators so that they increased in value as confidence increased. This allowed us to consider additive and multiplicative mechanisms as well as various 'voting' alternatives.

\subsection{Indirect Methods of Conveying Confidence}

It is worth mentioning that there are other more indirect ways of conveying confidence to the user. Rather than conveying confidence as a term or a numeric score it can be conveyed by giving the user some insight into the problem domain. Confidence can be conveyed by presenting explanation cases [12] or by highlighting whether a feature has a negative or positive correlation with respect to the classification [13] or by highlighting features that contribute positively and negatively to the classification [14] Confidence may also be conveyed by using visualisation tools to highlight features that contribute to similarity and to differences [15].

\section{Confidence Measures}

This section describes a number of confidence measures that could be used to predict confidence in ECUE, a case-based spam filter. We concentrate on using measures appropriate for a $k$-NN classifier. We evaluate these measures on a number of spam datasets to assess their performance at predicting confidence.

The $k$-NN measures that we propose evaluating, which are described in Section 3.1, perform some calculation on a ranked list of neighbours of a target case. We do not use the basic classification score of the target case as ECUE uses unanimous voting in the classification process to bias the classifier away

from FPs. Unanimous voting requires all the $k$ nearest neighbours retrieved to be of classification spam in order for the target case to be classified as spam. Therefore there is no classification 'score', as such.

\subsection{Proposed $k$-NN Confidence Measures}

The objective of the $k$-NN measures is to identify those cases that are 'close' (i.e. with high similarity) to cases of the same class as the target case and are 'far' (i.e. low similarity) from cases of a different class. The closer a target case is to cases from a different class, the higher the chance that the target case is lying near or at the decision surface. Whereas the closer a case is to other cases of the same class, the higher the chance that it is further from the decision surface.

All the confidence measures for $k$-NN discussed in this section use the ranked list of neighbours of a target case. This list of neighbours is a list of all the cases in the case-base ordered by distance from the target case. The measures can use 
- the distance between a case and its nearest neighbours (let $N N_{i}(t)$ denote the $i$ th nearest neighbour of case $t$ ) or,

- the distance between the target case $t$ and its nearest like neighbours (let $N L N_{i}(t)$ denote the $i$ th nearest like neighbour to case $t$ ) and/or

- the distance between a case and its nearest unlike neighbours (let $N U N_{i}(t)$ denote the $i$ th nearest unlike neighbour to case $t$ ).

The number of neighbours used in each measure is adjustable. All measures are constructed to produce a high score to indicate high confidence and a low score to indicate low confidence.

\section{Avg NUN Index}

The Average Nearest Unlike Neighbour Index (Avg NUN Index) is a measure of how close the first $k$ NUNs are to the target case $t$ as given in Equation 1.

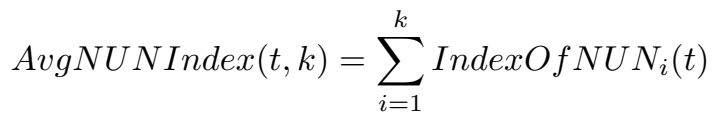

where IndexOfNUNi(t) is the index of the $i$ th nearest unlike neighbour of target case $t$.

This is illustrated in Figure 1 where for $k=1$ the index of the first NUN to target case $T_{1}$ is 5 whereas the index of the first NUN to target case $T_{2}$ is 2 , indicating higher confidence in the classification of $T_{1}$ than $T_{2}$.

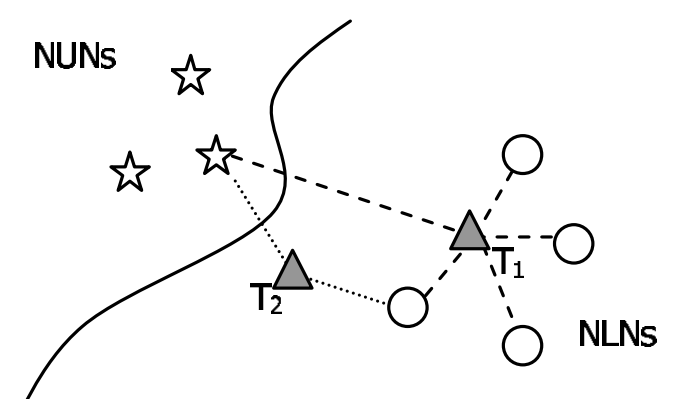

Fig. 1. Average NUN Index Confidence Measure

\section{Similarity Ratio}

The Similarity Ratio measure calculates the ratio of the similarity between the target case $t$ and its $k$ NLNs to the similarity between the target case and its $k$ NUNs, as given in Equation 2.

$$
\operatorname{SimRatio}(t, k)=\frac{\sum_{i=1}^{k} \operatorname{Sim}\left(t, N L N_{i}(t)\right)}{\sum_{i=1}^{k} \operatorname{Sim}\left(t, N U N_{i}(t)\right)}
$$


where $\operatorname{Sim}(a, b)$ is the calculated similarity between cases $a$ and $b$.

This is illustrated in Figure 2 where, for $k=1$, the similarity between the target case $T_{1}$ and its NLN is much higher than the similarity between $T_{1}$ and its NUN. Whereas the similarity between target case $T_{2}$ and its NLN is only marginally higher than the similarity between $T_{2}$ and its NUN. The ratio of these similarites for $T_{1}$ will give a higher result than that for $T_{2}$ indicating higher confidence in the classification of $T_{1}$ than $T_{2}$.

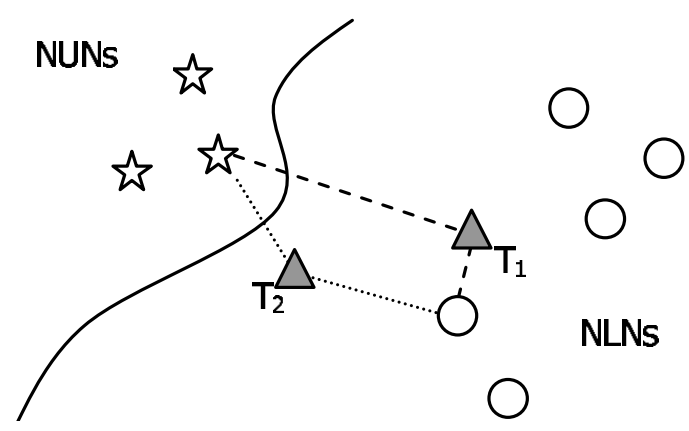

Fig. 2. Similarity Ratio Confidence Measure

\section{Similarity Ratio Within K}

The Similarity Ratio Within K is similar to the Similarity Ratio as described above except that, rather than consider the first $k$ NLNs and the first $k$ NUNs of a target case $t$, it only uses the NLNs and NUNs from the first $k$ neighbours. It is defined in Equation 3.

$$
\operatorname{SimRatio}(t, k)=\frac{\sum_{i=1}^{k} \operatorname{Sim}\left(t, N N_{i}(t)\right) 1\left(t, N N_{i}(t)\right)}{1+\sum_{i=1}^{k} \operatorname{Sim}\left(t, N N_{i}(t)\right)\left(1-1\left(t, N N_{i}(t)\right)\right)}
$$

where $\operatorname{Sim}(a, b)$ is the calculated similarity between cases $a$ and $b$ and $1(a, b)$ returns one if the class of $a$ is the same as the class of $b$ or zero otherwise.

This measure will attempt to reward cases that have no NUNs within the first $k$ neighbours, i.e. are in a cluster of $k$ cases of the same class. This is illustrated in Figure 3 where, considering $k=3$, the target case $T_{1}$ has no NUNs within the first three neighbours whereas target case $T_{2}$ has two NUNs and one NLN. The Similarity Ratio Within $\mathrm{K}$ will be much larger for $T_{1}$ than that for $T_{2}$ indicating higher confidence in the classification of $T_{1}$ than $T_{2}$.

If a target case $t$ has no NUNs then Equation 3 is effectively Equation 2 with the denominator set to one.

\section{Sum of NN Similarites}

The Sum of NN Similarities measure is the total similarity of the NLNs in the first $k$ neighbours of the target case $t$, see Equation 4 . 


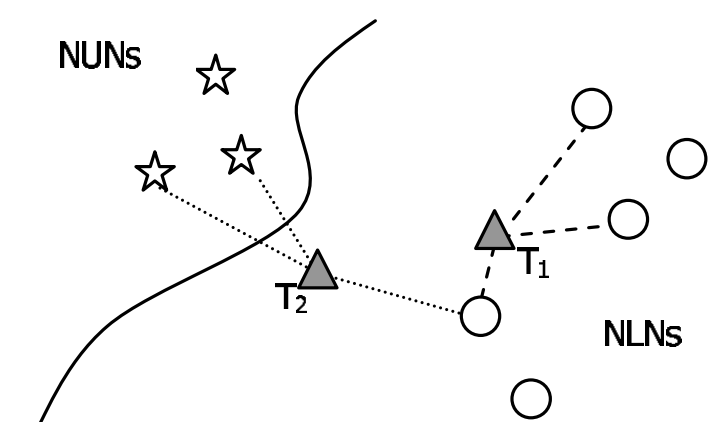

Fig. 3. Similarity Ratio Within K Confidence Measure

$$
\operatorname{SumNNSim}(t, k)=\sum_{i=1}^{k} 1\left(t, N N_{i}(t)\right) \operatorname{Sim}\left(t, N N_{i}(t)\right)
$$

where $\operatorname{Sim}(a, b)$ is the calculated similarity between cases $a$ and $b$ and $1(a, b)$ returns one if the class of $a$ is the same as the class of $b$ or zero otherwise.

For target cases in a cluster of cases of similar class this number will be large. For cases which are closer to the decision surface and have NUNs within the first $k$ neighbours, this measure will be smaller. In fact for target cases with no NUNs within the first $k$ neighbours this measure will be equal to the value of the Similarity Ratio Within K. Although this measure does not reward such cases as strongly as the Similarity Ratio Within K does as the resulting measure for the sum of the NLNs is not reduced by the influence of the NUNs.

\section{Average NN Similarity}

The Average NN Similarity measure is the average similarity of the NLNs in the first $k$ neighbours of the target case $t$, see Equation 5 .

$$
\operatorname{SumNNSim}(t, k)=\frac{\sum_{i=1}^{k} 1\left(t, N N_{i}(t)\right) \operatorname{Sim}\left(t, N N_{i}(t)\right)}{\sum_{i=1}^{k} 1\left(t, N N_{i}(t)\right)}
$$

where $\operatorname{Sim}(a, b)$ is the calculated similarity between cases $a$ and $b$ and $1(a, b)$ returns one if the class of $a$ is the same as the class of $b$ or zero otherwise.

\subsection{Assessing $k$-NN Confidence Measure Performance}

In order to assess the performance of these confidence measures we evaluated each of them on a number of spam datasets. The datasets are produced from the first stage of the case-base maintenance policy used by ECUE to handle concept drift in spam filtering [16]. ECUE's case-base maintenance procedure has two components; an initial case-base editing stage and a case-base update protocol. Each of the 5 datasets used is the result of performing the case editing procedure 
[17] on an initial case-base of 1000 emails (500 spam and 500 legitimate). After editing the datasets averaged 700 emails in size with an average of $45 \%$ spam and $55 \%$ legitimate emails.

The evaluation involved performing a leave-one-out validation on each dataset for each measure. We evaluated each measure using $k$ neighbours from $k=1$ upto $k=15$ and identified the confidence threshold, over all the $k$ values, that gave us the highest proportion of correctly predicted spam emails when there were no incorrect predictions (i.e. FPs). This is illustrated in Figure 4. We identified for each measure the value that maximised the number of spam correctly predicted with high confidence when the number of incorrect predictions with high confidence is zero.

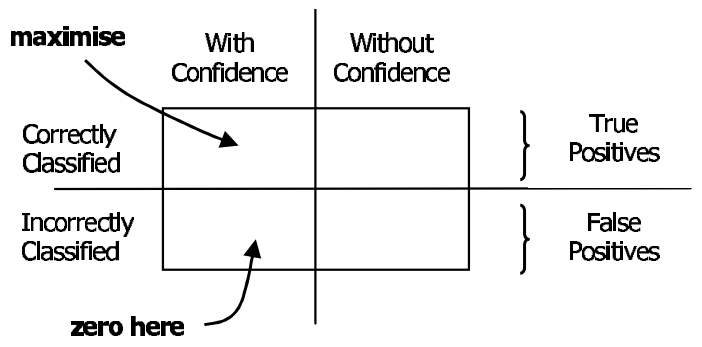

Fig. 4. Best Confidence Threshold Criteria

The results of this evaluation are presented in rows 1 to 5 of Table 1 . It details for each measure the highest percentage confidence that can be achieved on each dataset. This is the proportion of spam predictions that are made with high confidence. In all situations no highly confident incorrect predictions were made so no FPs are included in this proportion. In effect, this proportion of the spam can be ignored by the user, whereas the remaining percentage would have to be checked by the user.

Table 1. Best percentage confidence achievable for each dataset using different confidence measures

\begin{tabular}{|l|c|c|c|c|c|c|}
\hline & Dataset 1 & Dataset 2 & Dataset 3 & Dataset 4 & Dataset 5 & Avg \\
\hline Avg NUN Index & $23 \%$ & $76 \%$ & $75 \%$ & $41 \%$ & $44 \%$ & $51.8 \%$ \\
\hline Sim Ratio & $46 \%$ & $84 \%$ & $50 \%$ & $49 \%$ & $16 \%$ & $49.0 \%$ \\
\hline Sim Ratio Within k & $21 \%$ & $29 \%$ & $71 \%$ & $91 \%$ & $57 \%$ & $54.8 \%$ \\
\hline Sum NN Sim & $21 \%$ & $29 \%$ & $68 \%$ & $91 \%$ & $58 \%$ & $53.4 \%$ \\
\hline Avg NN Sim & $20 \%$ & $29 \%$ & $49 \%$ & $91 \%$ & $60 \%$ & $49.8 \%$ \\
\hline \hline Naive Bayes & $0 \%$ & $100 \%$ & $0 \%$ & $83 \%$ & $56 \%$ & $47.8 \%$ \\
\hline \hline ACM & $55.4 \%$ & $85.4 \%$ & $83.8 \%$ & $93.7 \%$ & $77.3 \%$ & $79.1 \%$ \\
\hline
\end{tabular}


Looking at the proportion of spam predictions for which confidence is high across all datasets it is evident that no single measure achieves good percentage confidence across all datasets. If we define "good" performance as having confidence in at least $50 \%$ of the spam predictions, none of the measures achieve "good" performance on more than three of the five datasets. The best performing measure is the Similarity Ratio Within K which has good performance on three of the five datasets with an average performance across all datasets of $54.8 \%$ but with minimum performance of $21 \%$.

\subsection{Naive Bayes Confidence Measure}

Naive Bayes is currently the machine learning technique of choise for spam filtering [18-20]. A Naive Bayes classifer also produces numeric scores, a 'probability' that can be used to predict confidence in the classifiers prediction. We evaluated using a Naive Bayes classifier on the 5 datasets. The implementation used is that described by Delany et al. [5]. The confidence threshold was identified as the highest numeric score returned by the classfier for a FP prediction. This ensured that no incorrectly classified spam emails were considered confident predictions. The 6 th row of Table 1 gives the confidence predictions for the 5 datasets using the Naive Bayes classifier. It is clear from the results that the Naive Bayes numeric score cannot be used as a predictor of confidence. In 2 of the 5 datasets there are zero confident predictions as there are FPs with the maximum score. In Dataset $2100 \%$ confidence is achieved as there were zero FPs in this dataset.

\subsection{Implications for Predicting Confidence in Spam Filtering}

To summarise, it appears that the confidence measures for $k$-NN and for Naive Bayes presented here can not consistently predict spam emails with good confidence. The average performance of the $k$-NN measures shows promise however the lack of consistency across all datasets is an issue. It is important to remember also that these figures are very optimistic as the test data is used to set the threshold.

\section{The Aggregated Confidence Measure}

Since none of the individual measures discussed in Section 3 was consistently effective at predicting confidence we evaluated a number of aggregation approaches which involved combining the results from the individual measures. We evaluated a number of aggregation approaches including the following:

(i) Summing the results from each of the 5 individual measures evaluated at the same value of $k$ and comparing the sum against a threshold;

(ii) Using the best threshold for each individual measure and indicating confidence if a certain number of the measures indicate confidence;

(iii) using a fixed $k$ across all measures and indicating confidence if a certain number of the measures indicate confidence. 
We found that the simplest and most effective method of aggregating the results is to assign confidence to a prediction if any of the individual measures indicated that the prediction was confident as in (ii) above. We call this measure the Aggregated Confidence Measure (ACM).

The algorithm for the ACM has two stages. First, identify the best threshold for each individual measure in the manner described in Section 3.2. A threshold consists of two values; the $k$ value indicating the number of neighbours to use, and the actual threshold value above which the prediction is considered confident. Then calcuate the score for each spam prediction against each individual measure using the appropriate threshold value of $k$ for the measure. If at least one of the scores for the individual measures is equal to or greater than the threshold value for that measure, confidence is expressed in the prediction.

\subsection{Assessment of ACM's Performance}

We evaluated the ACM on the 5 datasets already used in Section 3. The results are presented in row 7 of Table 1 . It is evident that the ACM is effective across all datasets with an average of $79 \%$ of the spam predictions being predicted with high confidence. The ACM also results in more than $50 \%$ of each dataset being predicted with high confidence. It is worth noting that the level of high confidence predictions for the ACM is also higher than the best individual measure's performance on each dataset (rows 1 to 5 of Table 1 ).

\subsection{Evaluation on Unseen Data}

One limitation of the evaluation performed in Section 4.1 is the that assessment was performed on the datasets which themselves were used to derive the confidence thresholds for the constituent confidence measures. In order to validate the ACM it is necessary to evaluate its performance on unseen data.

To do this we used ECUE along with two further datasets that have been used in concept drift evaluations of ECUE [16]. Each dataset is derived from an individual's mail received over the period of approximately one year. The first 1000 emails (consisting of 500 spam and 500 legitimate emails) in each dataset were used as training data to build the initial case-base classifier and the remaining emails were left for testing. To evaluate the ACM on unseen data involved building confidence thresholds for the ACM constituent measures on the initial case-base and then classifying the remaining emails using the ACM to determine how confident the spam predictions are. In this way, the test emails are not used in the determination of the confidence thresholds in any way.

The test emails were presented in date order for classification. Since this email data is subject to concept drift, ECUE's case-base update policy was applied to allow the classifier to learn from the new types of spam and legitimate email presented. The update policy has a number of components; an immediate update of the case-base with any misclassified emails when a FP occurred, a daily update of the case-base with any other misclassifieds emails that occurred that day, and a monthly feature reselection process to allow the case representation to take any 
new predictive features into account. In order to keep the confidence thresholds in line with the updates to the case-base an update policy for the confidence thresholds was also applied. This policy had two components; the confidence thresholds were updated whenever a confident FP email occurred and also after a monthly feature reselect.

Tables 2 and 3 show the results of testing the performance of the ACM on unseen data using the two datasets 6 and 7 . The tables present the accumulated monthly results for each dataset listing the total number and types of emails that were classified, the percentage of incorrect spam predictions (i.e. FPs) made (labeled \%FP classified) and the percentage of incorrect spam predictions made with high confidence (labeled \%Confident FPs). The table also gives the total percentage of spam predictions that had high confidence (labeled \%Confidence).

In both datasets predictions of confidence are high, averaging $85 \%$ in both cases with a lowest monthly level of $64 \%$. This is the percentage of spam predictions that can be ignored by the user, the remaining ones are either flagged in the Inbox as Maybe Spam or placed in a separate Maybe Spam folder for the user to check.

However in some of the months the ACM metric has resulted in confident incorrect predictions. Although the actual numbers of emails are low (four emails for Dataset 6 and six emails for Dataset 7) the ideal situation is one where all incorrect predictions have low confidence and will be flagged for the user to check. FPs flagged as confident will end up in the spam folder and may be missed by the user. Examining the confident FPs, three are emails from mailing lists and two are responses to Web registrations which users may not be too concerned with missing. The remaining five are important, some work related and one even a quotation in response to a online car hire request.

It is clear that we are approaching the limits of the accuracy of machine learning techniques in this domain. We see two possibilities for addressing these FPs. Close examination of such emails may identify domain specific characteristics that could be used as a feature or number of features in the case representation. Secondly, most deployed spam filtering solutions do not rely on one approach for filtering spam, they combine a number of techniques including white and black listing, rules, collaborative and learning approaches. Incorporating additional techniques into ECUE to add to its case-based approach could help in catching these outlier FPs.

\section{Conclusions}

We have shown that confidence measures based on the numeric scores from Naive Bayes or measures based on the $k$ nearest neighbours for a case-based classifier are not consistent at predicting confidence in the spam filtering domain. We have described an aggregation-based approach to combining individual confidence measures that shows great promise in confidently predicting spam. We evaluated this aggregated confidence measure by incorporating it into the classification process of a case-based spam filter and showed that it could successfully 
Table 2. Performance of ACM on unseen data using Dataset 6

\begin{tabular}{|l|c|c|c|c|c|c|c|c|c|}
\hline Month & $\mathbf{1}$ & $\mathbf{2}$ & $\mathbf{3}$ & $\mathbf{4}$ & $\mathbf{5}$ & $\mathbf{6}$ & $\mathbf{7}$ & $\mathbf{8}$ & Overall \\
\hline Total emails classified & 772 & 542 & 318 & 1014 & 967 & 1136 & 1370 & 1313 & 7382 \\
\hline Number of Spam & 629 & 314 & 216 & 925 & 917 & 1065 & 1225 & 1205 & 6496 \\
\hline Number of Non Spam & 93 & 228 & 102 & 89 & 50 & 71 & 145 & 108 & 886 \\
\hline \%FPs classified & $4.3 \%$ & $2.6 \%$ & $1.0 \%$ & $1.1 \%$ & $6.0 \%$ & $1.4 \%$ & $0.0 \%$ & $1.9 \%$ & $2.0 \%$ \\
\hline \%Confident FPs & $0.0 \%$ & $0.9 \%$ & $0.0 \%$ & $1.1 \%$ & $0.0 \%$ & $0.0 \%$ & $0.0 \%$ & $0.9 \%$ & $0.5 \%$ \\
\hline \%Confidence & $70 \%$ & $87 \%$ & $76 \%$ & $94 \%$ & $89 \%$ & $73 \%$ & $77 \%$ & $99 \%$ & $85 \%$ \\
\hline
\end{tabular}

Table 3. Performance of ACM on unseen data using Dataset 7

\begin{tabular}{|l|c|c|c|c|c|c|c|}
\hline Month & $\mathbf{1}$ & $\mathbf{2}$ & $\mathbf{3}$ & $\mathbf{4}$ & $\mathbf{5}$ & $\mathbf{6}$ & Overall \\
\hline Total emails classified & 293 & 447 & 549 & 693 & 534 & 495 & 3011 \\
\hline Number of Spam & 142 & 391 & 405 & 459 & 406 & 476 & 2279 \\
\hline Number of Non Spam & 151 & 56 & 144 & 234 & 128 & 19 & 732 \\
\hline \%FPs classified & $0.7 \%$ & $3.6 \%$ & $3.5 \%$ & $2.6 \%$ & $1.6 \%$ & $0.0 \%$ & $2.2 \%$ \\
\hline \% Confident FPs & $0.0 \%$ & $3.6 \%$ & $0.7 \%$ & $0.4 \%$ & $1.6 \%$ & $0.0 \%$ & $0.8 \%$ \\
\hline \%Confidence & $95 \%$ & $95 \%$ & $87 \%$ & $64 \%$ & $89 \%$ & $88 \%$ & $85 \%$ \\
\hline
\end{tabular}

separate the spam predictions into two sets, those with high confidence of spam which can be ignored by the user and those with low confidence which should be periodically checked for False Positives. The high-confidence set included $85 \%$ of the predicted spam reducing the number of spam that the user needs to check.

\section{References}

1. Mitchell, T.: Machine Learning. McGraw Hill, New York (1997)

2. Fausett, L.: Fundamentals of Neural Networks: Architectures, Algorithms, and Applications. Prentice Hall (1993)

3. Hosmer, D.W., Lemeshow, S.: Applied Logistic Regression. Wiley Series in Probability and Statistics. Wiley (2000)

4. Christianini, N., Shawe-Taylor, J.: An Introduction to Support Vector Machines: And Other Kernel-based Learning Methods. Cambridge University Press (2000)

5. Delany, S., Cunningham, P., Coyle, L.: An assessment of case-based reasoning for spam filtering. Artificial Intelligence Review (to appear) (2005)

6. Cheetham, W.: Case-based reasoning with confidence. In Blanzieri, E., Portinale, L., eds.: 5th European Workshop on Case-Based Reasoning. Volume 1898 of LNCS., Springer (2000) 15-25

7. Cheetham, W., Price, J.: Measures of solution accuracy in case-based reasoning systems. In Funk, P., González-Calero, P., eds.: 7th European Conference on CaseBased Reasoning (ECCBR 2004). Volume 3155 of LNAI., Springer (2004) 106-118

8. Lenat, D., Davis, R., Doyle, J., Genesereth, M., Goldstein, I., Schrobe, H.: Reasoning about reasoning. In Hayes-Roth, F., Waterman, D.A., Lenat, D.B., eds.: Building Expert Systems. Addison-Wesley, London (1983) 219-239 
9. Davis, R., Buchanan, B.: Meta level knowledge. In Hayes-Roth, F., Waterman, D.A., Lenat, D.B., eds.: Rule-Based Expert Systems. Addison-Wesley, London (1985) 507-530

10. Davis, R.: Expert systems: Where are we? and where do we go from here? AI Magazine 3 (1982) 3-22

11. McLaren, B.M., Ashley, K.D.: Helping a cbr program know what it knows. In Aha, D., Watson, I., eds.: 4th International Conference on Case-Based Reasoning (ICCBR-2001). Volume 2080 of LNAI., Springer (2001) 377-391

12. Doyle, D., Cunningham, P., Bridge, D., Rahman, Y.: Explanation oriented retrieval. In Funk, P., González-Calero, P.A., eds.: 7th European Conference on Case-Based Reasoning (ECCBR 2004). Volume 3155 of LNAI., Springer (2004) 157-168

13. Nugent, C., Cunningham, P.: A case-based explanation system for black-box systems. Artificial Intelligence Review (to appear) (2005)

14. McSherry, D.: Explaining the pros and cons of conclusions in cbr. In Funk, P., González-Calero, P., eds.: 7th European Conference on Case-Based Reasoning (ECCBR-2004). Volume 3155 of LNAI., Springer (2004) 317-330

15. Massie, S., Craw, S., Wiratunga, N.: A visualisation tool to explain case-base reasoning solutions for tablet formulation. In: 24th SGAI International Conference on Innovative Techniques and Applications of Artificial Intelligence (AI-2004). LNCS, Springer (2004)

16. Delany, S.J., Cunningham, P., Tsymbal, A., Coyle, L.: A case-based technique for tracking concept drift in spam filtering. Knowledge-Based Systems (to appear) (2005)

17. Delany, S.J., Cunningham, P.: An analysis of case-based editing in a spam filtering system. In Funk, P., P.González-Calero, eds.: 7th European Conference on CaseBased Reasoning (ECCBR 2004). Volume 3155 of LNAI., Springer (2004) 128-141

18. Androutsopoulos, I., J.Koutsias, Chandrinos, G., Paliouras, G., Spyropoulos, C.: An evaluation of naive bayesian anti-spam filtering. In Potamias, G., Moustakis, V., van Someren, M., eds.: Procs of Workshop on Machine Learning in the New Information Age, ECML 2000. (2000) 9-17

19. P.Pantel, Lin, D.: Spamcop: A spam classification and organisation program. In: Procs of Workshop for Text Categorisation, AAAI-98. (1998) 95-98

20. Sahami, M., Dumais, S., Heckerman, D., Horvitz, E.: A bayesian approach to filtering junk email. In: Procs of Workshop for Text Categorisation, AAAI-98. (1998) 55-62 